\title{
Reversible Posterior Leukoencephalopathy Syndrome in a Postpartum Woman without Eclampsia
}

\author{
Takeshi Uwatoko, Kazunori ToYodA, Yuko HiraI, Toshifumi SHIMADA, \\ Kotaro YASUMORI*, Setsuro IBAYASHI** and Yasushi OKADA
}

\begin{abstract}
We report a patient who developed reversible posterior leukoencephalopathy syndrome (RPLS) in puerperium without preeclampsia-eclampsia or chronic hypertension. The woman suddenly complained of visual loss and headache 10 days after delivery caused by edematous lesions mainly distributed in the bilateral occipital lobe. Apparent diffusion coefficient map was useful for distinction of this vasogenic edema from cytotoxic edema due to brain infarction. Under the diagnosis of RPLS, we successfully treated her disease using a trinitroglycerin as an antihypertensive, a hyperosmolar agent, methylprednisolone, and a free radical scavenger. Postpartum women may have the risk of development of RPLS even without preeclampsia-eclampsia. Vascular endothelial dysfunction may trigger RPLS, in addition to acute and modest increase in systemic pressure.

(Internal Medicine 42: 1139-1143, 2003)
\end{abstract}

Key words: brain edema, preeclampsia, hypertensive encephalopathy, apparent diffusion coefficient, free radical scavenger

\section{Introduction}

Reversible posterior leukoencephalopathy syndrome (RPLS), termed by Hinchey et al (1), has a unique neuroradiological finding of vasogenic edema in the brain and clinical symptoms including headache, altered mental function, seizure, and visual disturbance. Edema is predominant in the posterior portions of the white matter (1). Recent studies have also documented prevalent extension of edema into the brainstem and basal ganglia $(2,3)$. Hypertensive encephalopathy is the well-known cause (4), and renal decompensation, fluid retention, and the use of immunosuppressive drugs are other common etiologies (1). For pregnant and immediate postpartum women, eclampsia or preeclampsia is an important cause of RPLS. Preeclampsia is diagnosed on the basis of hypertension and proteinuria; edema has been removed in recent classifications as a defining sign of preeclampsia (5). Eclampsia additionally shows seizure. These disorders have been believed to share similar pathophysiologic mechanisms with hypertensive encephalopathy $(1,6)$. Here, we describe a patient who developed RPLS in puerperium without any indicators for preeclampsia-eclampsia in her pregnancy.

\section{Case Report}

A 30-year-old woman had a normal pregnancy and delivered normally her first baby at 38 weeks of gestation without proteinuria, peripheral edema, or neurological symptoms including seizure during pregnant period. Labor was induced with 20 units of oxytocin infusion. The total bleeding volume during delivery was normal $(186 \mathrm{ml})$. She did not have chronic hypertension and her blood pressure was consistently normal throughout her pregnancy; $105 / 69 \mathrm{mmHg}$ at 32 weeks and 130/65 mmHg at 38 weeks of gestation (Fig. 1). She was also normotensive during her hospitalization from the day of delivery to the 6th postpartum day. Ten days after the delivery (day 1), she had a headache in the morning, and when she woke up from a midday nap, she could not see anything. Her blood pressure was $152 / 104 \mathrm{mmHg}$ then. She was admitted to our cerebrovascular center immediately.

On arrival, she was alert and oriented. She barely distinguished brightness, and she could not distinguish any objects. She did not have any other neurological deficits. Her blood pressure was 220/90 $\mathrm{mmHg}$, pulse rate was $66 \mathrm{bpm}$ and regular, and her body temperature $37.0^{\circ} \mathrm{C}$. She did not

From the Department of Cerebrovascular Disease and *Neuroradiology, Cerebrovascular Center and Clinical Research Institute, National Kyushu Medical Center, Fukuoka and **Department of Medicine and Clinical Science, Graduate School of Medical Sciences, Kyushu University, Fukuoka

Received for publication April 21, 2003; Accepted for publication July 28, 2003

Reprint requests should be addressed to Dr. Kazunori Toyoda, the Department of Cerebrovascular Disease, National Kyushu Medical Center, 1-8-1 Jigyohama, Chuo-ku, Fukuoka 810-8563 


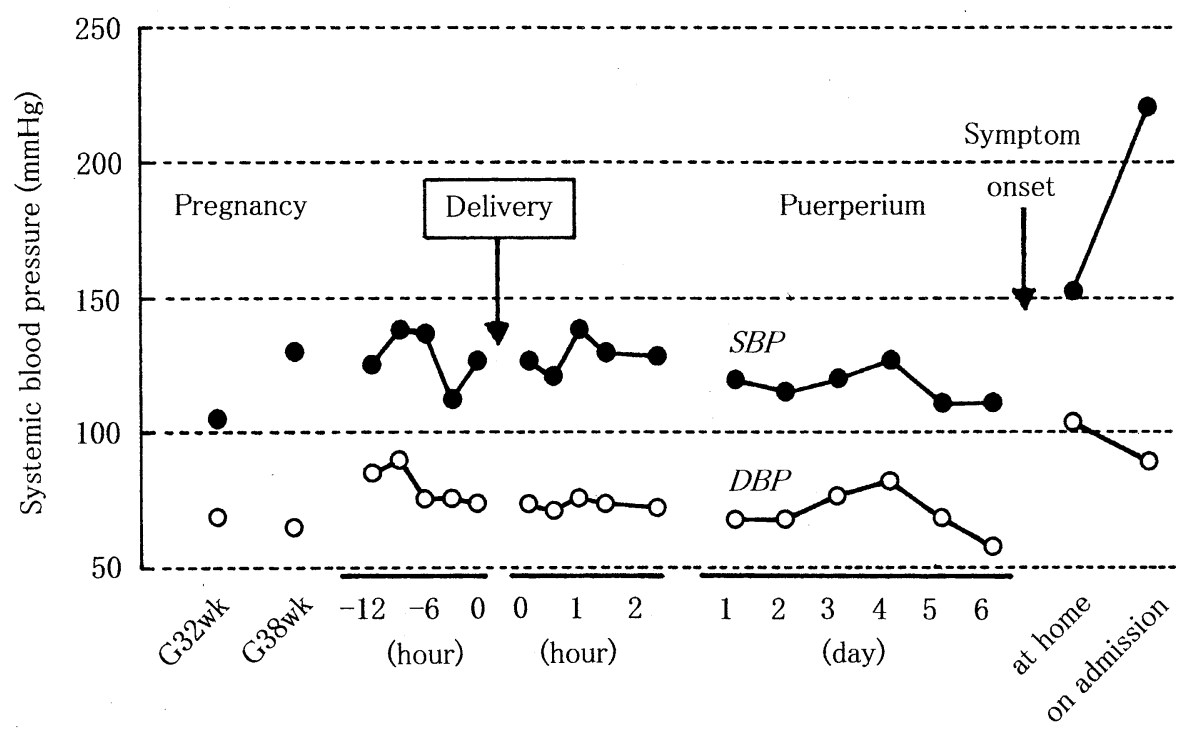

Figure 1. Change in systemic blood pressure during pregnancy, delivery, and puerperium. SBP: systolic blood pressure, DBP: diastolic blood pressure.

have heart murmur, respiratory rale, peripheral edema, or change in retinal arterioles and optic disc. Her urinalysis was normal. She had leukocytosis $\left(11,100 / \mathrm{mm}^{3}\right)$. Serum chemistry showed elevation of C-reactive protein (CRP, 1.69 $\mathrm{mg} / \mathrm{dl}$ ), lactic dehydrogenase (LDH, $331 \mathrm{IU} / \mathrm{l}$ ), and soluble vascular cell adhesion molecule-1 (sVCAM-1, $835 \mathrm{ng} / \mathrm{ml}$ ). Hemostatic tests, endocrinologic tests, and renin-aldosterone-angiotensin system were normal. Head CT revealed hypodense lesions in bilateral occipital lobes. Head MRI demonstrated identical lesions with CT. On fluid-attenuated inversion recovery (FLAIR), T2-, and diffusion-weighted images, hyperintense lesions expanded in bilateral occipital cortices and bilateral frontal cortices and subcortices (Fig. 2). The site of occipital lesions did not correspond with arterial territories, and thus brain infarction was not a possible etiology. Because cerebral venous thrombosis sometimes occurs in puerperium and has predominant lesions in the posterior portions of the brain, we immediately evaluated venous pathology using cerebral angiography, which did not reveal any vascular diseases including venous thrombosis and vasospasm. We diagnosed her occipital lesions as RPLS. We started intravenous infusion of trinitroglycerin to lower blood pressure to $<140 / 90 \mathrm{mmHg}$, glycerol to attenuate mass effect, and heparin to prevent from possible coagulopathy. Intravenous infusion of a free radical scavenger, edaravone, was also started because the agent might protect cerebral vascular endothelium against ischemic injury and inhibit vasogenic edema.

On the next morning (day 2), she could not see anything yet. On FLAIR, T2-, and diffusion-weighted images of 12hour follow-up MRI, hyperintense lesions increased especially in bilateral frontal lobes (Fig. 2). The apparent diffusion coefficient (ADC) in the lesions amounted to much higher value $\left(2.27 \times 10^{-3} \mathrm{~mm}^{2} / \mathrm{s}\right)$ than that of normal parenchymal tissue $\left(1.72 \times 10^{-3} \mathrm{~mm}^{2} / \mathrm{s}\right)$, suggesting vasogenic edema (Fig. 3). To prevent progression of edema, we added pulse therapy by drip intravenous infusion of methylprednisolone for three days, followed by oral prednisolone use. On the following day (day 3 ), she distinguished counting fingers, features, and large letters. By day 7, her visual function returned to normal. MRI-documented lesions shrunk on day 7 and disappeared on day 17 (Fig. 3). ADC values in the lesions were normal on days $7\left(1.75 \times 10^{-3} \mathrm{~mm}^{2} / \mathrm{s}\right)$ and $17(1.36 \times$ $\left.10^{-3} \mathrm{~mm}^{2} / \mathrm{s}\right)$.

\section{Discussion}

This is the first report on RPLS in puerperium without history of preeclampsia-eclampsia or other triggers including chronic hypertension, renal decompensation, fluid retention, and the use of immunosuppressive drugs.

An abrupt and severe increase in blood pressure, usually above the limit of diastolic pressure $>130 \mathrm{mmHg}$, is the leading mechanism of RPLS due to hypertensive encephalopathy $(7,8)$. Blood pressure at the onset of RPLS in eclamptic women was reported to be around $150-190 / 90-110 \mathrm{mmHg}$, and was not so high compared with that for hypertensive encephalopathy $(1,9,10)$. During preeclampsia-eclampsia, there is not sufficient time for an upper shift in the autoregulatory curve of cerebral blood flow. In such a circumstance, RPLS may occur during relatively acute but a quantitatively modest elevation of arterial pressure (11). An ultrasonographic study reported that, even under moderate elevation of systemic blood pressure, intracranial arterial blood flow velocities of preeclamptic/eclamptic women were pathologically increased (12). The present patient showed 

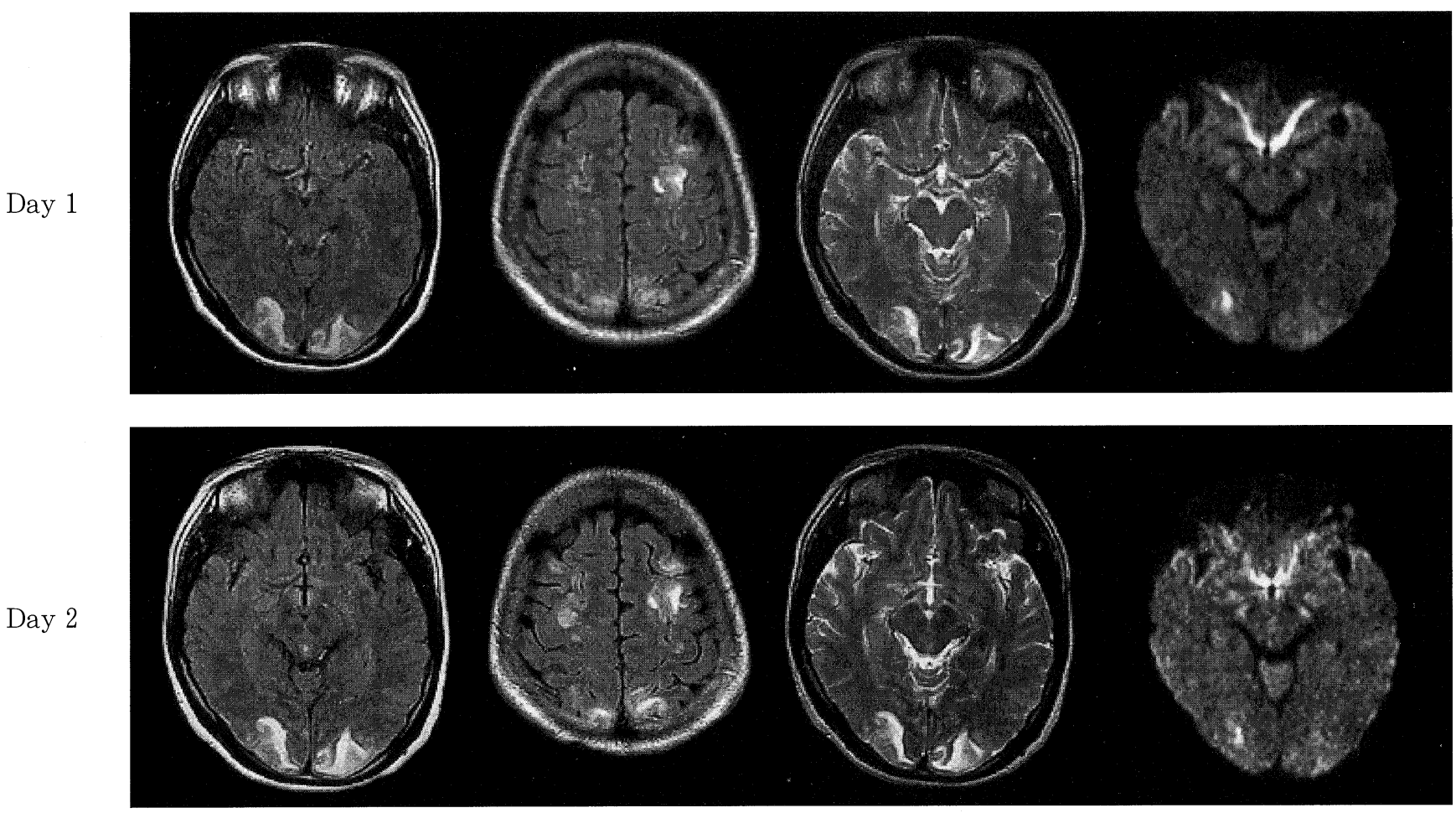

Figure 2. Head magnetic resonance imaging of the patient on days 1 and 2. On FLAIR, T2-, and diffusion-weighted images, hyperintense lesions expanded in bilateral occipital cortices and bilateral frontal cortices and subcortices. On day 2 , hyperintense lesions increased in bilateral frontal lobes.

higher blood pressure on admission (152/104 to 220/90 $\mathrm{mmHg}$ ) than usual, and it might augment progression of brain edema to some extent. The increase in blood pressure did not seem to result from the labor itself, because it was performed without vasoactive agents and without any troubles and the patient was normohypertensive at least for 6 days after the delivery.

In addition, endothelial dysfunction is considered to contribute to multiple-organ pathophysiology of preeclampsiaeclampsia (9, 13). Although pregnancy is associated with endovascular invasion of fetal trophoblast into maternal vessels and striking modifications of the spiral arteries that provide the blood supply to the placenta, these changes occur either very superficially or not at all in preeclampsia. The lack of normal changes in spiral arteries ends in abnormal placentation, placental ischemia, increase in circulating concentrations of numerous markers of endothelial activation including sVCAM-1, and alterations of endothelial function in multi-organ vessels (13). Thus, for preeclamptic women, disturbance of the blood brain barrier due to endothelial dysfunction in cerebral vasculature seems to be as an essential cause of RPLS as high blood pressure. As characteristic parameters in blood tests for RPLS in preeclampsia-eclampsia, abnormal red blood cell morphology and high levels of LDH were proposed (9). The present patient showed elevation of
LDH, although she did not have abnormal red blood cell morphology. In addition, she showed elevation of sVCAM1. This adhesion molecule from activated endothelial cells by inflammatory cytokines was reported to be increased in preeclampsia, but not in normal pregnancy and postpartum (14). Thus, although she was not clinically preeclamptic, she might have had tendency toward endothelial dysfunction in cerebral blood vessels. Elevation of CRP, leukocytosis, and mild fever up on admission suggested the existence of latent inflammation, and it may have triggered progression of endothelial dysfunction.

FLAIR images of MRI can detect subtle subcortical and cortical lesions in RPLS and are valuable for diagnosis in this disease $(2,15)$. In addition, high ADC values in this lesion could distinguish vasogenic edema in RPLS from cytotoxic edema due to brain infarction, carbon monoxide intoxication, and mitochondrial cytopathy. This discrimination is necessary for the determination of initial therapeutic strategies. Utility of ADC maps and diffusion-weighted imaging for diagnosis of RPLS was also ascertained in previous studies $(8,10,16)$.

Regarding therapeutic strategies, in addition to the basic therapy using antihypertensives and hyperosmolar agents, pulse therapy using methylprednisolone might be effective (17), because soon after beginning this therapy the condition 

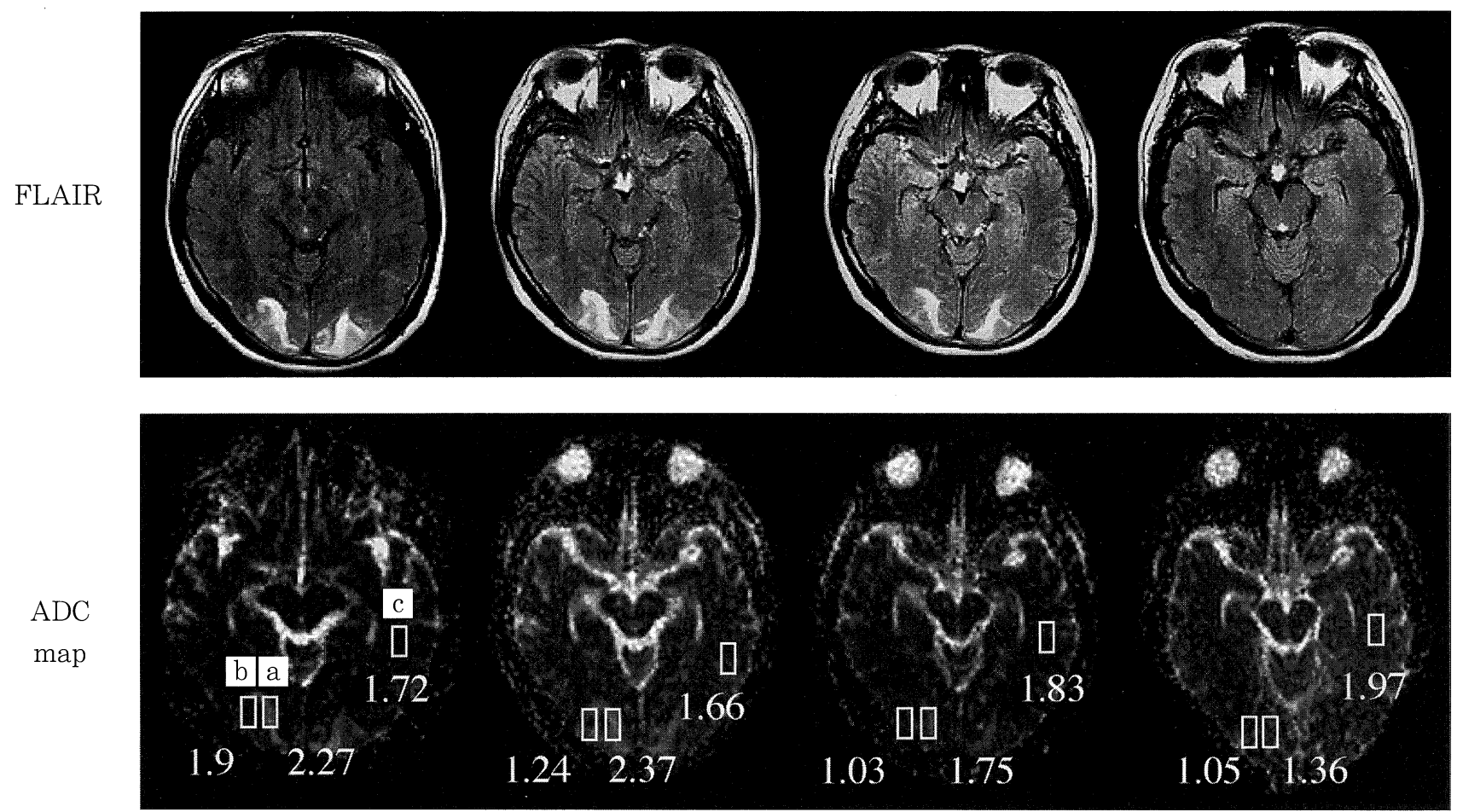

Figure 3. Head FLAIR images and ADC maps of the patient. ADCs of the edematous lesion (a), normal parenchyma near the lesion (b), and normal parenchyma in the contralateral hemisphere (c) were recorded. FLAIR-documented lesions shrunk on day 7 and disappeared on day 17. ADC of the lesion returned to normal on days 7 and 17.

of the present patient took a favorable turn. Because the free radical scavenger, edaravone (formerly MCI-186), prevents ischemic injury of vascular endothelial cell cultures (18), we used the agent to prevent vasogenic edema due to endothelial dysfunction. Further examinations seem to be needed for clinical evidence of usefulness of free radical scavengers for RPLS.

In conclusion, we successfully managed RPLS in a postpartum woman without preeclampsia-eclampsia. We should remember that RPLS can develop in puerperium without preeclampsia-eclampsia or chronic hypertension.

Acknowledgement: We thank Dr. Shin Nagata for his valuable advice. This study was partially supported by the Japanese Ministry of Health, Labor and Welfare (15C-1).

\section{References}

1) Hinchey J, Chaves C, Appignani B, et al. A reversible posterior leukoencephalopathy syndrome. N Engl J Med 334: 494-500, 1996.

2) Kumai Y, Toyoda K, Fujii K, Ibayashi S. Hypertensive encephalopathy extending into the whole brainstem and deep structures. Hypertens Res 25: 797-800, 2002

3) Chang GY, Keane JR. Hypertensive brainstem encephalopathy: three cases presenting with severe brainstem edema. Neurology 53: 652-654, 1999.

4) Vaughan CJ, Delanty N. Hypertensive emergencies. Lancet 356:
411-417, 2000.

5) Higgins JR, de Swiet M. Blood-pressure measurement and classification in pregnancy. Lancet 357: 131-135, 2001.

6) Dahmus MA, Barton JR, Sibai BM. Cerebral imaging in eclampsia: magnetic resonance imaging versus computed tomography. Am J Obstet Gynecol 167: 935-941, 1992.

7) Healton EB, Brust JC, Feinfeld DA, Thomson GE. Hypertensive encephalopathy and the neurologic manifestations of malignant hypertension. Neurology 32: 127-132, 1982.

8) Ay H, Buonanno FS, Schaefer PW, et al. Posterior leukoencephalopathy without severe hypertension: utility of diffusion-weighted MRI. Neurology 51: 1369-1376, 1998.

9) Schwartz RB, Feske SK, Polak JF, et al. Preeclampsia-eclampsia: clini$\mathrm{cal}$ and neuroradiographic correlates and insights into the pathogenesis of hypertensive encephalopathy. Radiology 217: 371-376, 2000.

10) Schaefer PW, Buonanno FS, Gonzalez RG, Schwamm LH. Diffusionweighted imaging discriminates between cytotoxic and vasogenic edema in a patient with eclampsia. Stroke 28: 1082-1085, 1997.

11) Heistad DD, Lawton WJ. Pathogenesis of acute hypertensive encephalopathy. In: Hypertensive Primer, second edition: The Essentials of High Blood Pressure, Izzo JL, Black HR, Eds. Lippincott, Williams \& Wilkins, Dallas, 1999: 186-187.

12) Zunker P, Ley-Pozo J, Louwen F, Schuierer G, Holzgreve W, Ringelstein EB. Cerebral hemodynamics in pre-eclampsia/eclampsia syndrome. Ultrasound Obstet Gynecol 6: 411-415, 1995.

13) Roberts JM, Cooper DW. Pathogenesis and genetics of pre-eclampsia. Lancet 357: 53-56, 2001.

14) Higgins JR, Papayianni A, Brady HR, Darling MR, Walshe JJ. Circulating vascular cell adhesion molecule-1 in pre-eclampsia, gestational hypertension, and normal pregnancy: evidence of selective 


\section{Reversible Postpartum Encephalopathy}

dysregulation of vascular cell adhesion molecule-1 homeostasis in preeclampsia. Am J Obstet Gynecol 179: 464-469, 1998.

15) Casey SO, Sampaio RC, Michel E, Truwit CL. Posterior reversible encephalopathy syndrome: utility of fluid-attenuated inversion recovery MR imaging in the detection of cortical and subcortical lesions. AJNR Am J Neuroradiol 21: 1199-1206, 2000.

16) Schwartz RB, Mulkern RV, Gudbjartsson H, Jolesz F. Diffusionweighted MR imaging in hypertensive encephalopathy: clues to pathogenesis. Am J Neuroradiol 19: 859-862, 1998.

17) Honkaniemi J, Kahara V, Dastidar P, et al. Reversible posterior leukoencephalopathy after combination chemotherapy. Neuroradiology 42: 895-899, 2000.

18) Watanabe T, Morita I, Nishi H, Murota S. Preventive effect of MCI186 on 15-HPETE induced vascular endothelial cell injury in vitro. Prostaglandins Leukot Essent Fatty Acids 33: 81-87, 1988. 